\title{
AFRL-RH-WP-TR-2014-0150
}

\section{Perception of Complex Auditory Scenes}

\author{
Eric Thompson and Billy Swayne
}

Ball Aerospace \& Technologies Corporation

Systems Engineering Solutions

Fairborn, OH 45324-6269

July 2014

Final Report

Distribution A: Approved for public release; distribution unlimited.

\section{STINFO COPY}

AIR FORCE RESEARCH LABORATORY

$7^{\text {th }}$ HUMAN PERFORMANCE WING

HUMAN EFFECTIVENESS DIRECTORATE

WRIGHT-PATTERSON AIR FORCE BASE, OH 45433-7334

AIR FORCE MATERIEL COMMAND

UNITED STATES AIR FORCE 


\section{Notice and Signature Page}

Using Government drawings, specifications, or other data included in this document for any purpose other than Government procurement does not in any way obligate the U.S. Government. The fact that the Government formulated or supplied the drawings, specifications, or other data does not license the holder or any other person or corporation; or convey any rights or permission to manufacture, use, or sell any patented invention that may relate to them.

Qualified requestors may obtain copies of this report from the Defense Technical Information Center (DTIC) (http://www.dtic.mil).

\section{AFRL-RH-WP-TR-2014-0150 HAS BEEN REVIEWED AND IS APPROVED FOR PUBLICATION IN ACCORDANCE WITH ASSIGNED DISTRIBUTION STATEMENT.}

//signed//

Brian D. Simpson, Ph.D.

Work Unit Manager

Battlespace Acoustics Branch //signed//

Robert C. McKinley

Chief, Battlespace Acoustics Branch

Warfighter Interface Division

//signed//

William E. Russell

Chief, Warfighter Interface Division

Human Effectiveness Directorate

$711^{\text {th }}$ Human Performance Wing

This report is published in the interest of scientific and technical information exchange and its publication does not constitute the Government's approval or disapproval of its ideas or findings. 


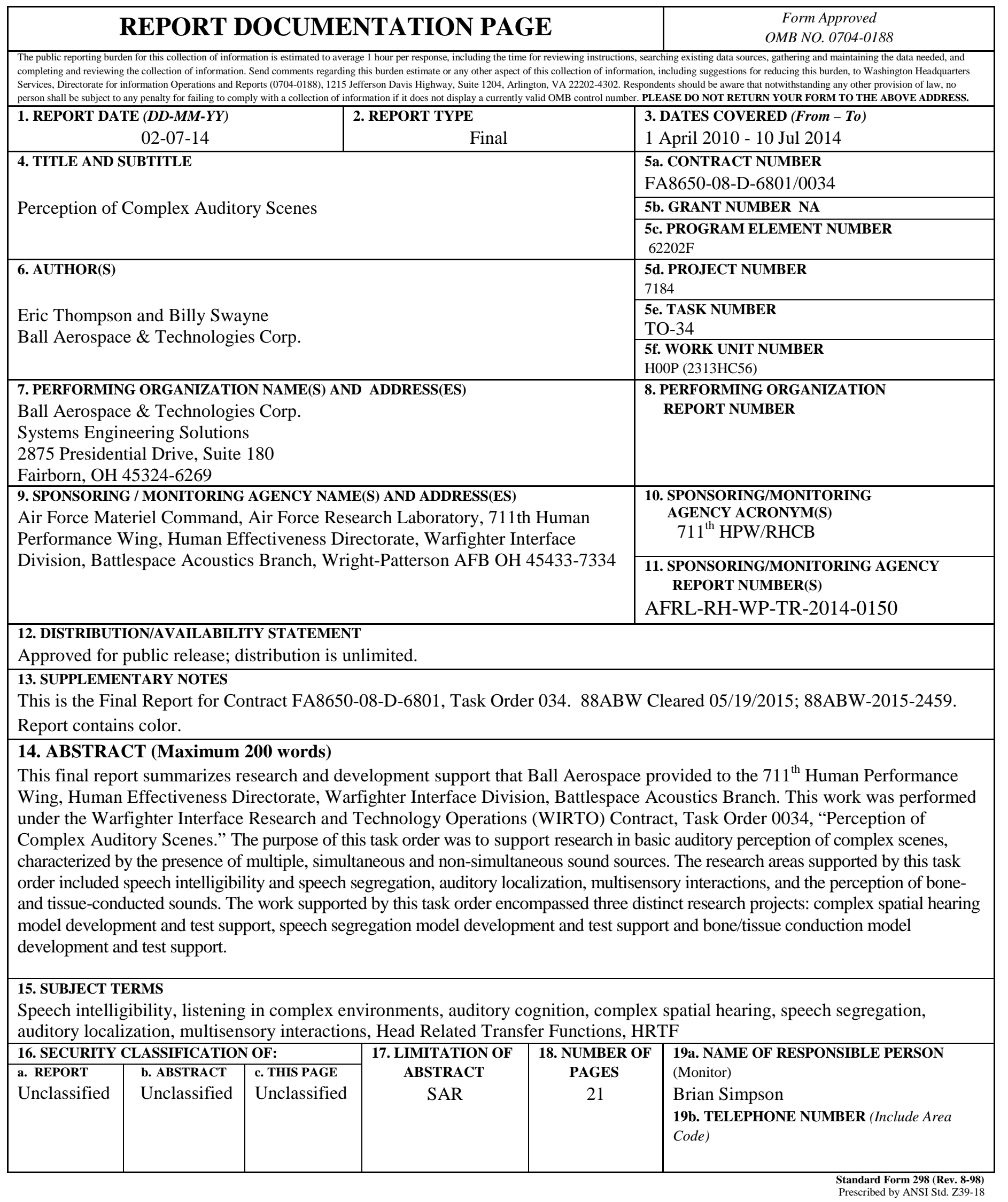


TABLE OF CONTENTS

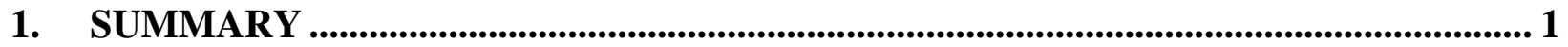

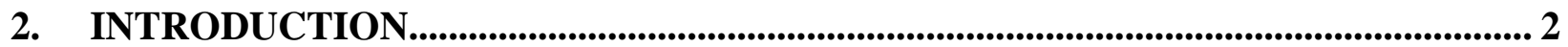

3. METHODS, ASSUMPTIONS, AND PROCEDURES .............................................. 3

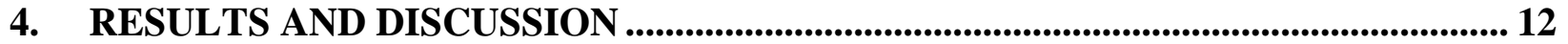

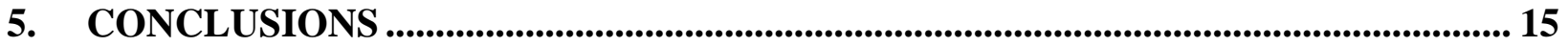

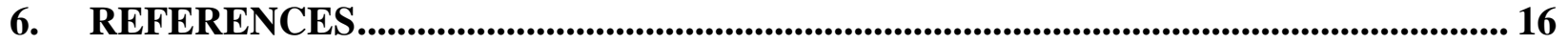

LIST OF SYMBOLS, ABBREVIATIONS, AND ACRONYMS....................................... 17 


\section{SUMMARY}

This final report summarizes research and development support that Ball Aerospace provided to the $711^{\text {th }}$ Human Performance Wing, Human Effectiveness Directorate, Warfighter Interface Division, Battlespace Acoustics Branch. This work was performed under the Warfighter Interface Research and Technology Operations (WIRTO) Contract, Task Order 0034, "Perception of Complex Auditory Scenes.” The purpose of this task order was to support research in basic auditory perception of complex scenes, characterized by the presence of multiple, simultaneous and non-simultaneous sound sources. The research areas supported by this task order included speech intelligibility and speech segregation, auditory localization, multisensory interactions, and the perception of bone- and tissue-conducted sounds. The work supported by this task order encompassed three distinct research projects: complex spatial hearing model development and test support, speech segregation model development and test support and bone/tissue conduction model development and test support.

Ball Aerospace met all task order requirements by providing scientific and technical support for testing, studies, and evaluations; research facility configuration and readiness; equipment management; and the management/coordination of subcontractors/consultants. In addition, engineering, consultant, experimental design and execution (including acoustic research subject provision and management), acoustic data collection and management, computer programming and analysis support were provided. All task objectives were met and required deliverables were submitted. 


\section{INTRODUCTION}

The purpose of the Warfighter Interface Research \& Technology Operations (WIRTO) contract (FA8650-08-D-6801) is to provide research operations for the Warfighter Interface Division ( $711^{\text {th }}$ HPW/RHC) at Wright-Patterson AFB, Ohio. Through this contract, Ball Aerospace supports the $711^{\text {th }}$ HPW/RHC's mission to conceive, develop, evaluate, integrate, and transition science and technology to revolutionize control, information availability, and cognitive interfaces in Air Force systems to enhance decision-making by commanders, operators, and support personnel. The WIRTO contract consists of numerous task orders (TOs) for providing a wide range of scientific and technical support for human performance research and technology enhancements.

As part of the $711^{\text {th }}$ HPW/RHC, the 711th Human Performance Wing's Battlespace Acoustics Branch (711 HPW/RHCB) has a long history of providing expert consultation and support to Air Force (AF), Department of Defense (DoD), and other government agency customers in the area of acoustics and auditory interface technologies. Three basic research projects addressed under TO-34 are Complex Spatial Hearing Model Development and Test Support, Speech Segregation Model Development and Test Support, and Bone Tissue Conduction Model Development and Test Support.

To perform such work, RHCB researchers required comprehensive scientific and technical support for testing, conducting studies and evaluations; research facility configuration and readiness; equipment management; and the management/coordination of subcontractors/consultants. In addition, engineering, consultant, experimental design and execution (including research subject provision and management), acoustic data collection and management, computer programming and analysis support were required.

Under TO-34, Ball Aerospace provided comprehensive support to RHCB to meet the above requirements. This TO comprised five subtasks: three technical subtasks, a program management subtask, and final report delivery. This report documents the activities and accomplishments of those subtasks and fulfills Contract Data Requirements List (CDRL) Data Item A001. 


\section{METHODS, ASSUMPTIONS, AND PROCEDURES}

The nature of the efforts specified in the Statement of Work (SOW) for Task Order 34 (TO-34) required that Ball Aerospace personnel work closely with on-site government personnel in executing the technical subtasks (Complex Spatial Hearing Model Development and Test Support, Speech Segregation Model Development and Test Support, and Bone Tissue Conduction Model Development and Test Support). Specific subtask activities varied in order to meet emerging requirements, and the focus of each subtask was determined through technical discussions among the Ball and Government Task Order Managers, and Ball and Government Principal Investigators. The subtasks were not entirely independent in the sense that the support required for one subtask sometimes also addressed the needs of another subtask.

A wide range of support was required in order to achieve the TO-34 objectives. It is not possible to describe all of these efforts in detail in a single report of reasonable size. In the discussion that follows, the requirements of each subtask are summarized, and examples of how Ball personnel supported the subtasks are provided.

To accomplish data collection in the following subtasks, a highly trained acoustic research subject panel, as well as ad hoc (on call) human test subjects participated in the studies and evaluations. No interaction with human research subjects commenced until approval from the Air Force Institutional Review Board (IRB) was obtained. Researchers followed all directives and regulations spelled out in the SOW (32 CFR 219, 45 CFR Part 46, DoD Directive 3216.02, Air Force Instruction 40-402, and AFRL Instruction 40-402) and abided by the Belmont Report for ethical treatment of human subjects. Hearing tests were administered to all members of the subject panel on a monthly basis to closely monitor their hearing. Depending on the nature of the evaluation, if a more sensitive hearing test was required, otoacoustic emissions tests were also conducted. Ball Aerospace holds a current Federal Wide Assurance (FWA).

\section{Complex Spatial Hearing Model Development and Test Support}

This subtask examined how sound sources combined in an auditory scene to form a context against which changes in spatial configuration of a target signal were perceived. The goal of this research was to develop and test models of spatial hearing in complex acoustic environments. (CDRL A004)

Ball personnel supported research studies in the Auditory Localization Facility (ALF). This facility is a 4.3-m diameter geodesic sphere housed in an anechoic chamber. 277 Bose 11-cm full -range loudspeakers are mounted on the surface of the sphere and a LED cluster is mounted at each loudspeaker location. Support for this subtask consisted of managing and scheduling 
acoustic subject panel members, operating and maintaining the facility and collecting data. Troubleshooting hardware/software problems in the ALF facility was also conducted, e.g., discovering a bad relay and fixing it as well as replacing the table response interface.

Ball provided assistance to the design of a mobile hearing lab for audiometric and localization screening. The design of a horizontal LED and speaker array and relay controller boards was completed and tested.

Additional support included measuring Head Related Transfer Functions (HRTFs), which are recordings that capture the direction-dependent acoustic transformations imposed on a sound by the pinna, head, and torso, of a listener. HRTFs are measured by placing miniature microphones in a subject's ear canals and recording stimuli from all of the speakers in ALF. The recordings are then rendered into an HRTF that allows the subject to hear in virtual 3D over a standard headset.

Some of the auditory localization studies that Ball Aerospace personnel supported are described below.

\section{Fringe_Exp2}

This experiment examined sound localization in the presence of a masker that may have up to $500 \mathrm{~ms}$ of forward masker fringe presented prior to the masker/signal interval. There was no auditory gap between the presentation of the fringe and the presentation of the masker/target. This experiment tested 'High Uncertainty' conditions only (i.e., the location of the fringe+masker stimulus varied from trial to trial).

\section{SpHarmOrderStudyInSituCombined}

This experiment was designed to determine what order of spherical harmonics best assists a listener's ability to localize. HRTFs were processed with seven different spherical harmonic orders, ranging from $2^{\text {nd }}$ order to $14^{\text {th }}$ order. Subjects completed a localization task in situ, post HRTF collection. They also completed a discrimination task in which they identified each stimulus as a "real" or "virtual" sound.

\section{ATSearch6_T}

This localization experiment examined how an audio cue and/or a tactile cue could aid during a visual search task. The subject was instructed to locate a target speaker in a field of distracters. Each speaker was equipped with a cluster of four LEDs. The target speaker was identified as having two or four LEDs lit in the cluster. The distracters had one or three LEDs lit. The subject visually searched for the target speaker and then identified it by entering a two or a four on the response wand. Subject performance was graded by duration of search time, with $100 \%$ 
accuracy. For one set of conditions, the subject was tasked with locating the target using a visual only search technique. For a second set of conditions, the subject had an audio cue co-located with the target speaker. For the third set of conditions, the subject had the assistance of a tactile vest. The tactile vest contained tactors positioned at 61 locations ( 1 tactor/location) across the torso. Each tactor was correlated with a set of speakers in the sphere. The subject felt a "buzz" from the tactor that was associated with the target speaker.

\section{SectoralHRTFLoc}

In an effort to determine how changing the number of spherical harmonics in a subject's HRTFs affects his/her ability to localize, personalized HRTFs were recorded for each subject using all 277 speaker locations in ALF. The HRTFs were processed using the following numbers of spherical harmonics: 2, 4, 6, and 8.

\section{MedianHRTFLoc}

This study was conducted to determine how changing the fidelity of a subject's HRTFs affected his/her ability to localize. Fidelity in this situation refers to the number of reference points used to create the HRTFs. Personalized HRTFs were recorded for each subject using all 277 speaker locations in ALF. The HRTFs were processed using the following number of real speaker locations as virtual reference points: $277,18,15,12,9,6,3$, and 0 . Subjects used a different subject's HRTF for the “ 0 ” condition.

\section{MBSMBDLoc}

This study was conducted to determine if spatial consistency could be used to identify and localize a target signal in a spatially varying background. Each trial consisted of a number of intervals, each interval containing 1 target noise burst (60 ms) and 1 or 2 masker noise bursts (also $60 \mathrm{~ms}$ ). The target was defined by a sequence of bursts that remained in the same spatial location for the duration across intervals, while the masker(s) bursts varied spatially across intervals. The number of intervals varied between 2 and 16 per trial. Each interval was presented in rapid succession.

\section{StinkBug4}

One target talker and two masking talkers were presented in a random spatial location along a $180^{\circ}$ arc. Subjects were asked to identify the information provided by the target talker by responding on a Graphic User Interface (GUI) on a touch screen laptop. The target talker and masking talkers were presented as either stationary or moving stimuli. The target talker and masking talkers' voices were also either fixed as one voice or mixed with several different voices for each block. Subjects were given either no cue, an audio cue (sound burst), or visual cue (lit LED) indicating the location of the target. 


\section{Peak Notch Removal Study}

HRTFs were collected for all subjects. A generic HRTF was generated using the Knowles Electronic Manikin for Acoustic Research (KEMAR). A series of composite HRTFs were created using different elements of each subject's individualized HRTF and the generic KEMAR HRTF. Stimuli were presented virtually over headphones to the subjects. The subjects were tasked to localize 2870 stimuli under the varying composite HRTF conditions to complete the experiment.

\section{PeakNotchBandStudy}

HRTFs were collected for each subject at the beginning of each half hour session. Stimuli were presented through rendered HRTFs to the subjects over near-field mounted ear buds. The fidelity of the HRTFs was altered by including and excluding varying frequency information. Roughly 20 hours of data collection was obtained for each subject.

\section{Speech Segregation Model Development and Test Support}

This second subtask examined cues that listeners utilized to segregate a target talker amidst multiple simultaneous interfering signals in detection, discrimination and identification listening tasks. The goal of this project was to develop a model of speech segregation.

(CDRL A004)

Ball Aerospace support for this subtask consisted of scheduling subjects, conducting literature searches and reviews, supporting experimental design, operating the facility, collecting and analyzing data and maintaining and repairing the facility as required.

The following studies were conducted in the Listening Laboratory, which is a facility within 711 HPW/RHCB consisting of sound-attenuating booths with headsets and computers for generating and presenting acoustic stimuli, controlling experiments, and providing instructions and a response interface for the subjects. The following summaries for the Broadbent studies and the SetSize study were taken from the 2012 Progress Report for "Speech Intelligibility in Complex Operational Environments,” F-WR-2002-0006-H.

\section{Broadbent Studies (Sidetone Pilot and Sidetone 7)}

It is often necessary for operational Air Force personnel to monitor and respond to multiple channels of ongoing radio communication at the same time. However, little is known about listeners' effectiveness at tasks that involve listening while speaking. Therefore, this research was aimed at examining listening effectiveness as well as the behavioral and cognitive outcomes in complex decision-making auditory tasks such as this. This study was adapted from Broadbent's (1952) classic study that demonstrated substantial interference, and subsequent loss of information, that occurred when participants were required to listen and talk at the same time. In 
order to evaluate listening effectiveness, listeners responded to a series of yes-no questions by a talker referring to a visual display on a computer monitor. The questions were highly structured and based on a typical radio-telephone procedure (e.g., "Hello Darkstar. This is Tango. Is there a cross on position five? Over."). Subjects were asked to respond using one of three possible response formats; long ("Hello Tango. This is Darkstar. Yes. Over."), medium ("Tango from Darkstar. Yes. Over."), or short ("Tango. Yes."). Listener-talker gender configurations and the rate of the incoming messages were varied. Response accuracy was evaluated based on whether the listeners assigned the response back to the call-sign of the talker posing the question, responded to the assigned listener call-sign and ignored messages addressed to a distracter callsign, as well as provided the appropriate yes/no response for the inquiry. Results revealed that rate changes had the largest impact on listeners' accuracy while gender configuration had little effect on performance. In addition, performance in the abbreviated response conditions resulted in fewer errors. Together these findings indicated that performance decrements are likely not the result of the incoming signal being masked at the periphery by the listeners' responses, but rather from lack of sufficient cognitive resources necessary to process incoming speech while simultaneously planning appropriate responses.

\section{SetSize}

This experiment evaluated a novel approach to assessing the relative benefits in speech intelligibility afforded by different types of maskers. An existing methodology uses adaptive tracking procedures to estimate the signal to noise ratio (SNR) required for a listener to achieve $50 \%$ correct performance (often referred to as the speech-reception threshold or SRT) for each individual masker condition, and compares the relative SNRs obtained in each. However, the fundamental assumption of this approach is that the underlying psychometric functions have roughly identical slopes, which is often violated with different types of maskers. Bernstein and Brungart (2011) proposed a new approach to evaluate effectiveness of different maskers based on the findings that word-identification performance improved with decreasing response set size. The idea was to test the two listener groups with different set sizes to yield performance that was equivalent across groups. SRTs for different maskers measured, using these two different set sizes for the two groups, could then be examined to evaluate group differences without the confound of differences in underlying psychometric functions. This assumption was tested by measuring nonsense-syllable identification for normal-hearing listeners as a function of SNR, set size, and masker (stationary noise, 4- and 32-Hz modulated noise, and an interfering talker). These results, along with those of a second experiment evaluating an adaptive set-size algorithm to adjust performance levels, established set size as an efficient and effective tool to adjust baseline performance, when comparing effects of masker fluctuations between listener groups. 


\section{CRM_dichotic experiment}

The following abstract is from Thompson, E. R., Iyer, N., Simpson, B. D., \& Romigh, G., (2014). "Ear dominance in a dichotic cocktail party." Journal of the Association for Research in Otolaryngology, Abstract 37, p 518.

Cherry (1953) reported that when listeners were presented with a dichotic signal over headphones, they could reliably report words presented to the attended ear with very few errors, while only being aware of gross properties of the talker in the unattended ear. More recently, Gallun et al. (2007) showed that there were large differences in performance on a dichotic listening task depending on the ear of presentation. The number of errors was significantly larger when the target was presented to the left rather than to the right ear. Phrases selected from the Coordinate Response Measure (CRM) corpus were presented to listeners over headphones in three listening conditions : a) Target+Noise presented to left or right ear with no contralateral masker; b) Target+Noise presented to either ear with noise in the contralateral ear; and c) Target+Noise presented to either ear with a contralateral Speech+Noise masker, where the speech masker is a different, same-sex talker CRM phrase. Other independent variables were target ear (left or right), type of cueing (target ear precue vs. postcue), type of listening condition within a block (random ear-of-presentation vs. fixed), and signal-to-noise ratio (-18 to $-6 \mathrm{~dB}$ in 3-dB steps). The dependent variable was percent correct target color-number identification. Listeners showed ear dominance in that target identification was significantly better when the target signal was presented in the right ear than in the left ear. The right ear advantage was only evident in conditions with a contralateral speech masker, and was strongest in the postcue condition. When two speech inputs are presented simultaneously, the signal in the right ear appears to be preferentially processed. This preferential processing is present when the target ear is fixed throughout a block of trials, stronger when the target ear is randomly selected and cued before each trial, and strongest when the target ear is cued after each trial.

\section{ContralateralContext Experiment}

The following summary of this experiment was obtained from the 2013 Progress Report for Understanding the Relative Salience of Acoustic Cues in Speech Perception, F-WR-2012-0165H.

The goal of this experiment was to understand the cues that listeners use to determine which time-frequency (T-F) regions belong to the target and/or masker speech. To answer the question, several studies were conducted in order to determine what binauralintegration cues listeners use to segregate a target speech when it is masked by a spatially-separated fluctuating masker. The experiments measured psychometric 
functions for word-recognition performance in the presence of stationary noise, modulated noise, and one or two same- or opposite-gender interfering talkers. In order to evaluate the relative importance of the cues, the type of signal that was presented to the two ears was manipulated. In all experiments, the first ear received an unprocessed mixture containing the target and masker(s). The second ear received: a) no signal, b) an unprocessed mixture containing just the maskers, or c) mixture containing just the maskers that were processed with an eight-channel noise vocoder. The results showed that spatial release from masking occurred when an unprocessed mixture containing the maskers were presented for all conditions for all masker types, but that it only occurred in the vocoded condition with only same-gender interfering talkers. Results also revealed that listeners could segregate the target with as few as two vocoder channels, and that maximum performance occurred with six or more channels. These results suggest that speech intelligibility for a target signal can be improved in situations where monaural cues are insufficient for concurrent speech-stream segregation by providing even a degraded copy of the maskers to the opposite ear.

\section{Spatial Field and Dual Task Experiments}

These two experiments were designed to measure the listening effort required to understand speech in noise. An auditory dual-task design was used to stress the limits of auditory cognition, assuming that as the effort required to perform the primary task increased, performance on the secondary task would decrease. The primary task in both experiments was to identify a spoken color-number pair (e.g., red-seven) presented from a position in front of the listener. The secondary task was a critical call sign detection task, in which the listener had to detect when a call sign from a list of one or four call signs was presented from a randomly selected position in the horizontal plane in front of the listener. In the spatial field experiment, the cognitive load on the primary task was varied by increasing how many items the listener had to maintain in memory when the targets were presented in quiet. In the dual task experiment, the interaction between sensory load and cognitive load was investigated by varying both the signal-to-noise ratio and the memory load independently. The data showed that the cognitive load has a large impact on performance. The performance on the secondary task decreased greatly as the primary task difficulty increased, but the sensory and cognitive loads appeared to have independent effects on difficulty.

\section{Dicho_Detect Experiment}

The dicho_detect experiment investigated informational masking in speech detection, discrimination, and identification by measuring each task with different kinds of ipsilateral and contralateral maskers (noise, speech, time-reversed speech) as a function of target-to-masker ratio (TMR). The thresholds for identification were higher than those for discrimination for all conditions, and detection thresholds were the lowest for all conditions. Speech maskers raised 
thresholds the most, particularly when both maskers were speech. Time-reversed speech and noise maskers had similar effects on thresholds, indicating that these maskers were mostly energetic in nature.

\section{Bone and Tissue Conduction Model Development and Test Support}

This third subtask was to investigate the relative contributions of bone conduction and tissue conduction to loudness judgments, hearing damage risk criteria, and auditory localization. The purpose of this line of research was to develop and improve models of bone and tissue conduction and guide the development of effective hearing protection systems for high-noise environments. (CDRL A004)

As of the time of this report, work on this subtask has not yet commenced, due to lack of resources. This project will begin once this issue is resolved.

\section{Subcontractor Support Activities}

Ball's WIRTO partners on this task order were: JXT Applications, Inc., Dr. David Kieras, Dr. Greg Wakefield, and John Stewart. JXT Applications provided consistent access to paid volunteer talkers and listeners for the conduct of psychoacoustic listening tasks requiring them to detect, localize, identify, or classify auditory stimuli presented over headphones or via loudspeakers in 711 HPW/RHCB laboratories. Dr. David Kieras and Dr. Greg Wakefield developed additional components for the Executive-Process/Interactive Control (EPIC) model for communication and audition. In addition, they tested and validated the developed EPIC model with ongoing performance data obtained from RHCB. John Stewart supported the development of prototype software to support basic research in the perception of complex scenes, characterized by the presence of multiple, simultaneous and non-simultaneous sound sources.

\section{Final Report}

This final report documents the support provided by Ball Aerospace personnel and the task results. It was prepared in Air Force Technical Report format for publication, and submitted to the designated Air Force point of contact for review and revision before the final version was submitted as a contract deliverable. (CDRL A001)

\section{Program Management}

In support of this effort, Ball's Program Management Team performed a number of functions to ensure that the technical effort was performed and managed within the funding scope and 
schedule. The Program Manager and supporting management, financial, and administrative personnel developed and submitted a number of task-specific Contract Data Requirements List (CDRL) items. These included a Monthly Funds and Man-Hour Expenditure Report (FMHR), a Quarterly Contract Funds Status Report (CFSR), and a Monthly Project Status Report (MSR). Additional deliverable-related activities included the review and delivery of Briefing Materials, an Interim Report, and the project Final Report.

In addition to deliverable development, the Program Manager performed a number of functions to coordinate the technical activities over the course of the effort. These included holding periodic meetings with the Technical Monitor, the Contracting Officer's Technical Representative (COTR), the Ball technical team, and the subcontractors. Program management activities also included task planning, resource management and subcontractor management activities to ensure that $711 \mathrm{HPW} / \mathrm{RHCP}$ was receiving the support required throughout the life of the program. (CDRL's A002, A003 \& A004) 


\section{RESULTS AND DISCUSSION}

Ball Aerospace met all task order requirements by providing scientific and technical support for auditory testing, research studies, and evaluations; research facility configuration, readiness and repair; equipment management; and the management/coordination of the acoustic subject panel, subcontractors and consultants. Ongoing support was provided to research planning, experimental design, data analysis, publication of results, modeling and programming. All task objectives were met and required deliverables were submitted.

Numerous abstracts, papers, proceedings and poster presentations were prepared, in collaboration with colleagues from RHCB and other institutions, and submitted to conferences and meetings.

- Four abstracts were written and submitted to the Acoustical Society Meeting in Montreal with colleagues from RHCB, Wright State University, University of Maryland and Walter Reed, NMMC:

Thompson, E. R., Simpson, B. D., \& Iyer, N. (2013). Multicomponent signal detection: Tones in noise and amplitude modulation detection. Journal of the Acoustical Society of America, 133(5), p. 3285.

Iyer, N., Thompson, E. R., Simpson, B. D., Brungart, D. S., \& Summers, V. (2013). Exploring auditory gist: Comprehension of two dichotic, simultaneously presented stories. Journal of the Acoustical Society of America, 133(5), p. 3513.

Brungart, D., Iyer, N., Thompson, E., Simpson, B. D., Gordon-Salant, S., Shurman, J., Vogel, C., \& Grant, K. W. (2013). Interactions between listening effort and masker type on the energetic and information masking of speech stimuli. Journal of the Acoustical Society of America, 133(5), p. 3435.

Simpson, B., Gilkey, R., Thompson, E., Brungart, D., Iyer, N., \& Romigh, G. (2013). Spatial consistency as a cue for segregation and localization. Journal of the Acoustical Society of America, 133(5), p. 3510.

- Four papers were written with colleagues from RHCB and Walter Reed NMMC and submitted to the ICA/ASA 2013 conference in Montreal, QC. Papers were published in the Proceedings of Meetings on Acoustics (POMA):

Iyer, N., Thompson, E. R., Simpson, B. D., Brungart, D. \& Summers, V. (2013). Exploring auditory gist: Comprehension of two dichotic, simultaneously presented stories. Proceedings of Meetings on Acoustics (POMA), 19, 050158.

Brungart, D., Iyer, N., Thompson, E. R., Simpson, B. D., Gordon-Salant, S., Schurman, J., Vogel, C., \& Grant, K. (2013). Interactions between listening effort and masker type on the energetic and information masking of speech stimuli. Proceedings of Meetings on Acoustics (POMA), 19, 060146. 
Thompson, E. R., Iyer, N., Simpson, B. D. (2013). Multicomponent signal detection: Tones in noise. Proceedings of Meetings on Acoustics (POMA), 19, 050030.

Simpson, B. D., Gilkey, R., Thompson, E. R., Brungart, D., Iyer, N., \& Romigh, G. (2013). Spatial consistency as a cue for segregation and localization. Proceedings of Meetings on Acoustics (POMA), 19, 050140.

- Two abstracts and posters were prepared with colleagues from RHCB and Walter Reed NMMC and presented at the $33^{\text {rd }}$ meeting of the Association for Research in Otolaryngology in Baltimore, MD:

Summers, V., Thompson, E., Iyer, N., \& Brungart, D. (2013). Effects of glottal phase on negative level effects ("rollover") in speech perception. Journal of the Association for Research in Otolaryngology, Abstract 36, p. 106.

Iyer, N., Thompson, E., Romigh, G., Brungart, D., \& Simpson, B. (2013). Effects of target and masker spatial continuity on speech intelligibility. Journal of the Association for Research in Otolaryngology, Abstract 36, p. 254.

- An abstract and presentation were prepared with colleagues from RHCB and Wright State University, which was presented at the $165^{\text {th }}$ meeting of the Acoustical Society of America in San Francisco, CA:

Gilkey, R. H., Simpson, B. D., Thompson, E. R., Iyer, N., \& Romigh, G. (2013). Temporal effects when localizing targets defined by spatial consistency: Relation to Hafter's work on "binaural adaptation.” Journal of the Acoustical Society of America, 134(5), p. 4165.

- Two abstracts and posters were prepared with colleagues from RHCB and presented at the $34^{\text {th }}$ meeting of the Association for Research in Otolaryngology in San Diego, CA:

Thompson, E., Iyer, N., Simpson, B. D., \& Romigh, G. (2014). Ear dominance in a dichotic cocktail party. Journal of the Association for Research in Otolaryngology, Abstract 37, p. 518.

Wisniewski, M., Thompson, E., Iyer, N., Simpson, B., \& Sullivan, S. (2014). Oscillatory dynamics of EEG correlate with build-up in an informational masking task. Journal of the Association for Research in Otolaryngology, Abstract 37, p. 54.

- Four abstracts and posters were prepared with colleagues from RHCB, Wright State University, George Mason University, and Walter Reed NMMC and presented at the $166^{\text {th }}$ meeting of the Acoustical Society of America in Providence, RI:

Simpson, B. D., Gilkey, R., Iyer, N., Thompson, E., Romigh, G., \& Brungart, D. (2014). The effect of masker spatial uncertainty on sound localization. Journal of the Acoustical Society of America, 135(4), p. 2283. 
Thompson, E. R., Iyer, N., Romigh, G. D., \& Simpson, B. D. (2014) Mapping spatial release from informational masking with one or two masker talkers. Journal of the Acoustical Society of America, 135(4), p. 2281.

Iyer, N., Thompson, E. R., Romigh, G. D., Baldwin, C. L., \& Simpson, B. D. (2014). Spatial attention in an auditory dual task. Journal of the Acoustical Society of America, 135(4), p. 2280.

Romigh, G. D., Simpson, B. D., Thompson, E. R., \& Iyer, N. (2014). Relative importance of individual spectral features for intraconic localization. Journal of the Acoustical Society of America, 135(4), p. 2190.

- An abstract was prepared with colleagues from RHCB and submitted to the Cognitive Hearing Science for Communication conference to be held in June in Linkoping, Sweden.

- Assistance was provided with writing LRIR proposals based on the Dual-Task experiment and on the cognitive modeling of simultaneous speakers.

- The final report was written for 711 HPW Chief Scientist Seedling Grant for Multicomponent Signal Detection.

- A paper was written with colleagues from RHCB and the University of Michigan on a twotalker experiment and cognitive modeling:

Wakefield, G. H., Kieras, D., Thompson, E., Iyer, N., Simpson, B. D. (2014). EPIC modeling of a two-talker CRM listening task. The $20^{\text {th }}$ International Conference on Auditory Display (ICAD), June 22-25, New York, NY.

- A paper was written with colleagues from RHCB and the University of Michigan on speech perception with two simultaneous talkers and the development of a cognitive model that predicts human performance in that task:

Kieras, D. E., Wakefield, G. H., Thompson, E., Iyer, N., Simpson, B. D. (2014). A cognitive architectural account of two-channel speech processing. International Annual Meeting of the Human Factors and Ergonomics Society (HFES), October 27-31, Chicago, IL. 


\section{CONCLUSIONS}

TO-34 was accomplished according to the requirements of the SOW. All requirements were met. All issues were resolved. Subcontractor efforts were managed and coordinated to produce desired results. All deliverables were submitted. Ball Aerospace believes that the Air Force customer was pleased with the support and the results. 


\section{REFERENCES}

2012 Progress Report for “Speech Intelligibility in Complex Operational Environments,” F-WR2002-0006-H.

Broadbent, D. E. (1952). Speaking and listening simultaneously. Journal of Experimental Psychology, 43(4), 267-273.

Bernstein, J. G. W. \& Brungart, D. S. (2011). Effects of spectral smearing and temporal finestructure distortion on the fluctuating-masker benefit for speech at a fixed signal-to-noise ratio. Journal of the Acoustical Society of America, 130, 473-488.

Thompson, E. R., Iyer, N., Simpson, B. D., \& Romigh, G. (2014). "Ear dominance in a dichotic cocktail party." Journal of the Association for Research in Otolaryngology, Abstract 37, p 518.

Cherry, E. C. (1953). Some experiments on the recognition of speech with one and two ears. Journal of the Acoustical Society of America, 25, 975-979.

Gallun, F. J., Mason, C.R., \& Kidd, Jr., G. (2007). Task-dependent costs in processing two simultaneous auditory stimuli. Perception \& Psychophysics, 69(5), 757-771.

2013 Progress Report for "Understanding the Relative Salience of Acoustic Cues in Speech Perception,” F-WR-2012-0165-H. 


\section{LIST OF SYMBOLS, ABBREVIATIONS, AND ACRONYMS}

3D

$711^{\text {th }} \mathrm{HPW} / \mathrm{RHCB}$

AFRL

ALF

BATC

CDRL

CFSR

CRM

DoD

EPIC

FMHR

FWA

GUI

HRPP

HRTF

IRB

KEMAR

LED

MMC

MSR

RHCB

SNR

SOW

SRT

$\mathrm{T}-\mathrm{F}$

TO

WIRTO

WPAFB
Three Dimensional

Air Force Research Laboratory, Human Performance

Wing, Human Effectiveness Directorate, Warfighter Interface Division, Battlespace Acoustics Branch

Air Force Research Laboratory

Auditory Localization Facility

Ball Aerospace \& Technologies Corp.

Contract Data Requirements List

Contract Funds Status Report

Coordinate Response Measure

Department of Defense

Executive-Process/Interactive Control

Funds and Man-hours Expenditure Report

Federal Wide Assurance

Graphic User Interface

Human Research Protection Program

Head Related Transfer Function

Institutional Review Board

Knowles Electronic Manikin for Acoustic Research

Light Emitting Diode

Multi-Modal Communication

Monthly Status Report

Human Effectiveness Directorate-Warfighter Interface

Division, Battlespace Acoustics Branch

Signal to Noise Ratio

Statement of Work

Speech Reception Threshold

Time-Frequency

Task Order

Warfighter Interface Research \& Technology Operations

Wright-Patterson Air Force Base 